\title{
Are Artificial Neural Networks Useful for Predicting Overhanging Dental Restorations? A Cross-sectional Study
}

\author{
Hani T Fadel ${ }^{1}$, Osama Abu-Hammad ${ }^{2}$, Omar A Ghulam ${ }^{3}$, Najla Dar-Odeh ${ }^{4}$
}

\begin{abstract}
Aims: To predict the number of overhanging dental restorations (ODRs) using an artificial neural network (ANN) and determine the most important predictive variables.

Materials and methods: Patient- and restoration-related data were used as input variables to construct two networks, with (network 1) and without (network 2) the number of secondary caries lesions (SCLs) as input data. Output data were the number of ODRs. Of the 502 participants, data of the first 100 were used to build/train the model. Those of the remaining 402 were used to test the model for prediction accuracy.

Results: Model accuracy notably increased after training. Prediction of ODRs was more accurate in network 1 . Allowing for an error of \pm 1 , network 1 predicted the number of ODRs with an accuracy of $85.6 \%$, whereas that of network 2 was only $82.1 \%$ accurate. The number of old fillings was the most important input variable, while gender was the least important.

Conclusion: Within the study limits, the ANN model predicted ODRs with more than $85 \%$ accuracy. The number of old fillings was the most important predictive variable.

Clinical significance: Making use of ANN analyses can help periodontists and general dentists predict the occurrence of ODRs, formulate effective treatment planning, and reduce patient discomfort and unnecessary costs.

Keywords: Algorithms, Dental restoration failure, Neural networks, Overhanging dental restoration, Prediction.

World Journal of Dentistry (2020): 10.5005/jp-journals-10015-1709
\end{abstract}

\section{INTRODUCTION}

Dental restorations or tooth fillings are customized threedimensional additions to teeth, which can be made out of different materials and serve different clinical situations. ${ }^{1}$ They form a mere replacement of the missing tooth structure rather than treatment for the multifactorial disease process of dental caries. ${ }^{2}$ The quality of the dental restoration may be impacted by factors related to the patient, the dentist, and/or the restoration itself. ${ }^{3}$

Once in place, dental restorations may suffer from a number of mechanical or biological complications. ${ }^{4}$ These have adverse effects on the surrounding structures and can jeopardize the success of the restoration, leading to increased patient discomfort and wasting of resources. ${ }^{5}$ Overhanging dental restorations (ODRs) are noted as extensions of the restorative material beyond the confines of the cavity preparation. ${ }^{6}$ Overhanging dental restorations may be associated with bleeding, gingivitis, and periodontal tissue destruction. ${ }^{6}$ Possible reasons behind ODRs include inadequate use of matrix band system, suboptimal isolation, and/or improper handling of used restorative material.

Several mathematical models have been proposed to predict disease processes and complications in an attempt to actively prevent them and minimize treatment expenses. Artificial neural networks (ANN) are data-driven tools, used to analyze complex relationships. Unlike other models, data in ANN-based models are not fit to certain predefined conditions (e.g., linearity, normal distribution). Instead, experimental models are developed following training of available data. ${ }^{7}$ Usually, when the expert's knowledge in a certain area is not substantial, ANN modeling may be of particular use. It works by adjusting of weights between the neurons for any input-output function approximation. ${ }^{8}$ In dentistry, ANN has been applied in predicting the occurrence of dental pain, ${ }^{9}$ recurrent aphthous ulcerations, ${ }^{10}$ tooth surface loss, ${ }^{11}$ and several others.
${ }^{1}$ Department of Preventive Dental Sciences, Taibah University Dental College and Hospital, Madinah, Kingdom of Saudi Arabia

${ }^{2}$ Department of Substitutive Dental Sciences, Taibah University Dental College and Hospital, Madinah, Kingdom of Saudi Arabia; Department of Removable Prosthodontics, School of Dentistry, University of Jordan, Amman, Jordan

${ }^{3}$ Department of Dental Services, Prince Mohammed Bin Abdulaziz Hospital, National Guard Health Affairs, Madinah, Kingdom of Saudi Arabia

${ }^{4}$ Department of Oral and Basic Clinical Sciences, Taibah University Dental College and Hospital, Madinah, Kingdom of Saudi Arabia; Department of Oral Surgery, Oral Medicine and Periodontics, School of Dentistry, University of Jordan, Amman, Jordan

Corresponding Author: Hani T Fadel, Department of Preventive Dental Sciences, Taibah University Dental College and Hospital, Madinah, Kingdom of Saudi Arabia, Phone: +966 55440 6095, e-mail: hani.fadel@yahoo.com

How to cite this article: Fadel HT, Abu-Hammad O, Ghulam OA, et al. Are Artificial Neural Networks Useful for Predicting Overhanging Dental Restorations? A Cross-sectional Study. World J Dent 2020;1 1(2):99-104. Source of support: Nil

Conflict of interest: None

To date, no studies have managed to predict the occurrence of ODRs mathematically to avoid associated periodontal tissue destruction, patient overtreatment, and unnecessary treatment costs. The study objectives were thus to predict the occurrence of ODRs using an ANN mathematical model in individuals with approximal dental restorations and to determine the most important predictive variables according to the model. 


\section{Materials and Methods}

\section{Study Design and Sample}

This study was a cross-sectional, analytic investigation. It was conducted at Prince Mohammed Bin Abdulaziz Hospital, one of the tertiary care centers of the Ministry of the National Guard in AlMadinah AlMunawwarah, Kindom of Saudi Arabia. It regularly receives dental referrals from multiple primary care satellite clinics in the region. The study population comprised patients who visited the dental department for any form of dental care. All visiting patients routinely undergo a standard radiographic examination. Periapical and bitewing images were taken using a ROMIX 3.6.0.R radiographic unit (Planmeca Oy, Helsinki, Finland) and a digital prosensor (Planmeca ProSensor HD digital sensor system) via paralleling technique with short cones (16"). Exposure time was set at 0.50 seconds for posterior and anterior teeth, and the image density was fixed at $6 \mathrm{~mA}$ and $63 \mathrm{kV}$. Films were placed with standardized devices (Planmeca Trollbyte Plus). Inclusion criteria were patients who had approximal dental restorations in one or more posterior teeth as shown on the radiographs. Out of 1,388 visiting patients, only 502 (36\%) fitted the criteria at the time of the study.

\section{Study Data}

Demographic data and health status were collected from the patients' electronic medical record. Caries experience for each patient according to age was designated as low, normal, or high. ${ }^{12}$ Moreover, the type of approximal dental restoration and the date of the procedure were recorded. Two key parameters were observed from the radiographic images of all 20 premolars and molars:

- Overhanging dental restorations: "An extension of restorative material of any type beyond the confines of a cavity preparation." ${ }^{\prime 6}$

- Secondary caries lesions (SCLs): "A radiolucent area underlying a restoration and resembles the radiographic appearance of dental caries." 13

The same examiner assessed all images to reduce interexaminer variability.

\section{Data Analysis}

Means and standard deviations were used to describe quantitative data, while frequency distributions and percentages were used for qualitative data. The IBM ${ }^{\circledR}$ SPSS $^{\circledast}$ statistical analysis software version 20.0 (IBM, Armonk, New York, USA) was used.

\section{ANN Modeling}

The ANN modeling software (Pythia $\odot$; Runtime Software, USA) was run on a standard personal computer (Windows 10॰ 2017; Microsoft Corporation). This software employs backpropagation networks that calculate the differences between the net predicted and actual output values and propagates the difference (error) back to the output. In a neural network, two phases are distinguished: a training phase and a reproduction phase. During the training phase, training data containing both inputs and actual outputs are processed to optimize the network's output by minimizing the deviation according to the following equation: ${ }^{14}$

$$
\text { (Output } \left._{\text {Data }}-\text { Output }_{\text {Net }}\right)^{2}
$$

where Output $_{\text {Data }}$ is the output value in the training data and Output $_{\text {Net }}$ is the output value provided by reproducing the input data by the network.
This optimization is carried out by changing the synaptic weights. The network optimizes the output by continuously changing synaptic weights until it meets-ideally - the actual output. Synaptic weights are initially given a preliminary value of 0.5 . Each neuron's output is calculated according to the following equation:

$$
\mathrm{O}_{\mathrm{n}}=\mathrm{F}\left(\sum_{\mathrm{k}} \mathrm{I}_{\mathrm{k}} * \mathrm{~W}_{\mathrm{kn}}\right)
$$

where $O$ is the neuron's output, $n$ is the number of the neuron, $I_{k}$ is the neurons inputs, $k$ is the number of inputs, $W_{\mathrm{kn}}$ is the neurons' synaptic weights, $F$ is the Fermi function $1 /\left(1+\operatorname{Exp}\left(-4^{*}(x-0.5)\right)\right)$.

During the reproduction or testing phase of the analysis, unknown input data are fed to the network, and the network produces the output.

The ANN modeling software normalizes input (independent variables) and output data once the data have been introduced to the program by noting the minimum and maximum values for each variable. It also allows for setting the limits of normalization for each variable by the operator. Normalization is a process of expressing the values of each variable as a range from 0 to 1 . Independent variables included age, gender, medical status, caries experience, number of double-sided proximal fillings, number of old dental fillings, number of new dental fillings, number of temporary fillings (TFs), number of glass ionomer cement (GIC) fillings, number of amalgam fillings, number of composite fillings, number of crown retainers/abutments, number of bridge pontics, and number of SCLs. The limits for each of the tooth-related variables were set according to the minimum and maximum possible values per tooth and/or tooth surfaces of the 20 evaluated posterior teeth and as shown on the two-dimensional bitewing radiographs.

\section{Model Buildup}

Data from the first 100 participants were used to build up and train the ANN, while the remainder of the data, i.e., $n=402$, were kept for testing the network. The ANN software (Pythia; Runtime Software) used input and output data for the first 100 participants to suggest a number of ANNs through a genetic algorithm module (Fig. 1) and assigned each network with an accuracy card ranging from 0 to $100 \%$. An appropriate network (network 1) was chosen with the following criteria: accuracy card of $100 \%$, two-layer architecture, a number of neurons in the first layer matching the number of input data for the network, and a single neuron in the second layer. After the network was constructed, output data for the test sample were reproduced (ODRs - net output). This output was saved for later analysis. The network was further trained for 50,000 cycles to yield higher accuracy. After training, the output was reproduced again (ODRs — net after training). Values were again saved for later analysis, and the optimized trained network was thus ready for testing and production of ODR predictions.

Another network (network 2) was constructed in the same way but after omitting SCLs as an input variable. The network was also a two-layer network but with 13 neurons in the first layer to match the number of input data and 1 in the second. The network was optimized in the same way as network 1.

\section{Model Testing and Prediction of ODRs}

Data from the remaining participants $(n=402)$ were used to test the network by feeding in their input data, i.e., independent variables to the network. The optimized, trained network then reproduced a net output for this set of undisclosed (testing) data. This was saved 


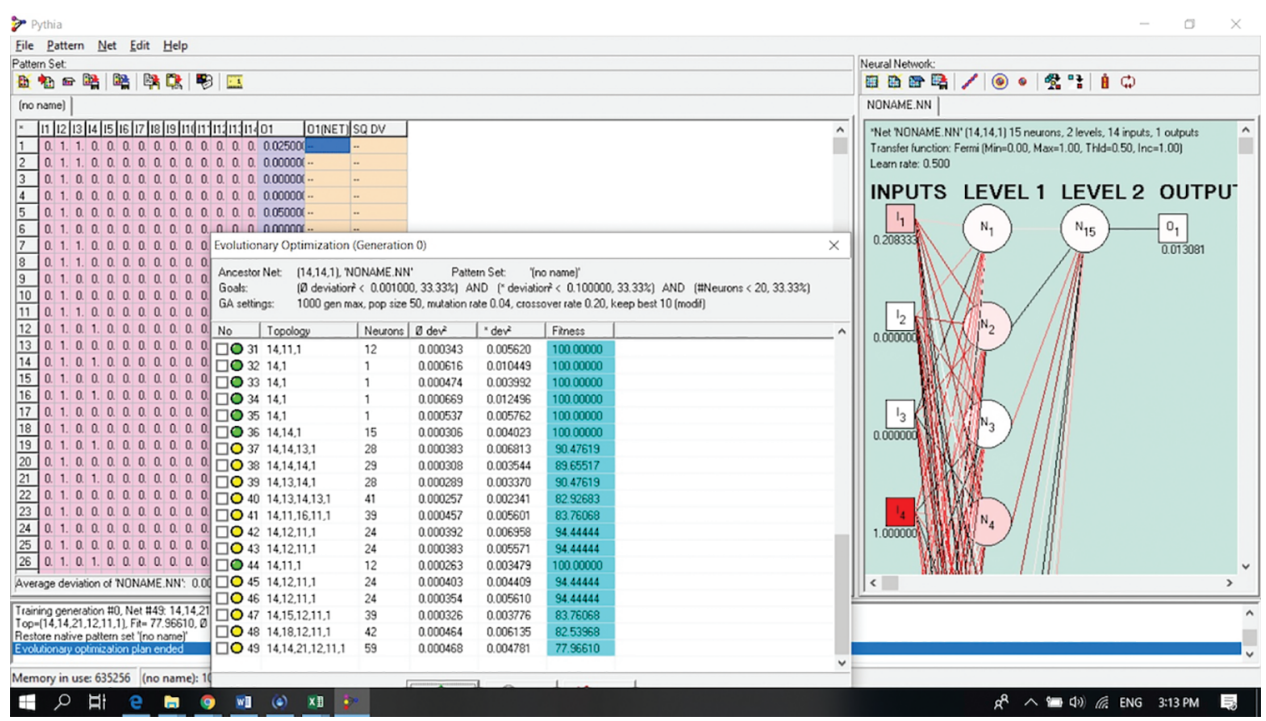

Fig. 1: Screenshot image of the graphical user interface of the Pythia software showing the genetic algorithms module in the foreground, input data in the background, and an artificial neural network on the right. The data column $\mathrm{O}_{1}$ on the far right is the net prediction for the number of overhanging dental restorations

as predictions of ODRs of network 1. Network 2 was also used to obtain predictions of ODRs that were saved for later analysis.

\section{Determining Factor Importance}

Data from the total sample $(n=502)$ were fed into the IBM SPSS statistical analysis software version 20.0 (IBM) to construct a multilayer perceptron network, similar to network 1 in topology. This was performed since the ANN modeling software could not provide information on the importance of the different variables contributing to the predictions. Secondary caries lesions were included as input data. All default settings within the software were accepted as such, and the analysis was conducted accordingly.

\section{Ethical Considerations}

Identities and personal information were kept confidential. Obtained information was only to be used for educational and research purposes. Patients were contacted about any needs for further treatment based on the radiographic study findings. Study approval was obtained from the Prince Mohammed Bin Abdulaziz Hospital Administration Committee (MAD-16-057550-123878, Reg. date August 3,2016). A request for waiver of consent was also approved by the administration committee given the nature of the study.

\section{Results}

The mean age of the 502 included participants was $38 \pm 13$ years (range: 15-87 years), 252 (50\%) of which were females. Sixty-nine (14\%) participants reported suffering from at least one medical condition. A high caries experience was observed in $28 \%$ of the sample, while normal caries experience was reported in $61 \%$.

Tables 1 and 2 show topology and synaptic weights of the two constructed networks after training [see eqn (2)].

The accuracy of predicting ODRs was demonstrated in both networks (Table 3). Network 1 showed a prediction accuracy of $86 \%$ when allowing for a \pm 1 margin of error. However, this prediction accuracy was reduced to $82 \%$ in network 2 when SCLs were removed from the set of input data.
Figure 2 shows the average deviations of the two networks when predicting ODRs after every 10,000 training cycles up to 50,000 cycles. The figure demonstrates that the more the training, the less the deviations.

The relative importance of input data, i.e., independent variables, in network 1 was also evaluated and illustrated (Fig. 3). The number of old fillings in the mouth showed the highest importance (100\%), while gender had the least (11\%).

\section{Discussion}

The aim of the current study was to predict the occurrence of ODRs in hospital attendees with approximal dental restorations. With selected patient- and restoration-related variables, the ANN model managed to predict the occurrence of ODRs with an accuracy of over $85 \%$. This was in line with the high accuracy of ANN models reported in the available dental literature. ${ }^{15}$ In a model developed for the prediction of toothache, Kim et al. reported a predication accuracy of $80 \% .{ }^{9}$ Similarly, Dar-Odeh et al. achieved an accuracy of $91 \%$ for predicting recurrent aphthous ulcerations. ${ }^{10}$ Such findings confirm the usefulness of ANN usage in dentistry and motivate further research and application.

The developed ANN utilized a number of input data to predict ODR occurrence, the highest importance of which being the number of old fillings in an individual. This may be understandable since amalgam, for example, is known to creep with time, ${ }^{16}$ while composite undergoes shrinkage, ${ }^{17}$ both of which leading to considerable changes in the restoration architecture and consequent accumulation of plaque and food debris. ${ }^{3}$ In such instances, and when adding suboptimal restorative clinical measures such as infrequent use of dental wedges, a restoration overhang might be inevitable.

The occurrence of ODRs has also been claimed to be related to diet. ${ }^{18,19}$ Moradi-Lakeh et al. reported the consumption of considerable amounts of energy drinks following a large national survey in Saudi Arabia. ${ }^{20}$ This might suggest an association with the occurrence of ODRs, as fizzy drinks cause tooth erosion, which may in turn contribute to an overhanging filling. However, dietary 


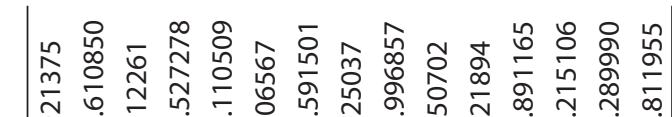

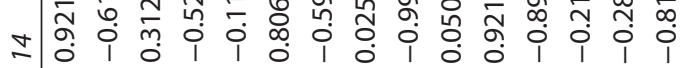
○

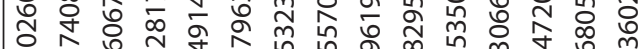
m $\uparrow$ i o i

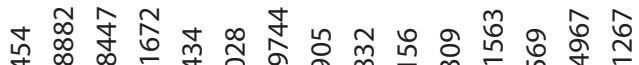

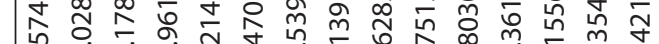

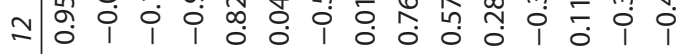
๒

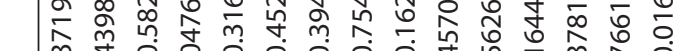

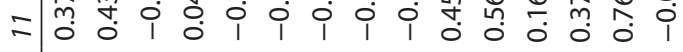

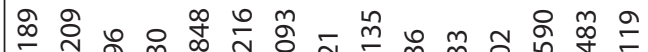

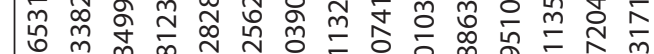

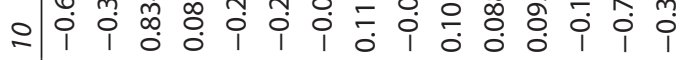

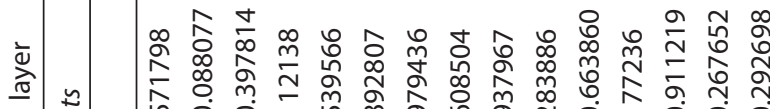

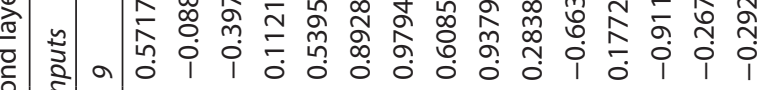

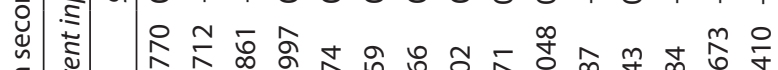

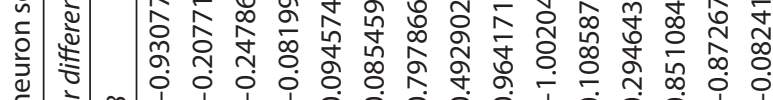

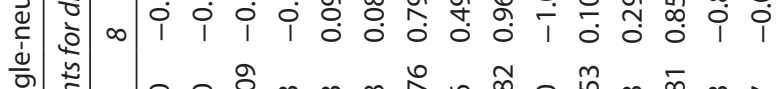

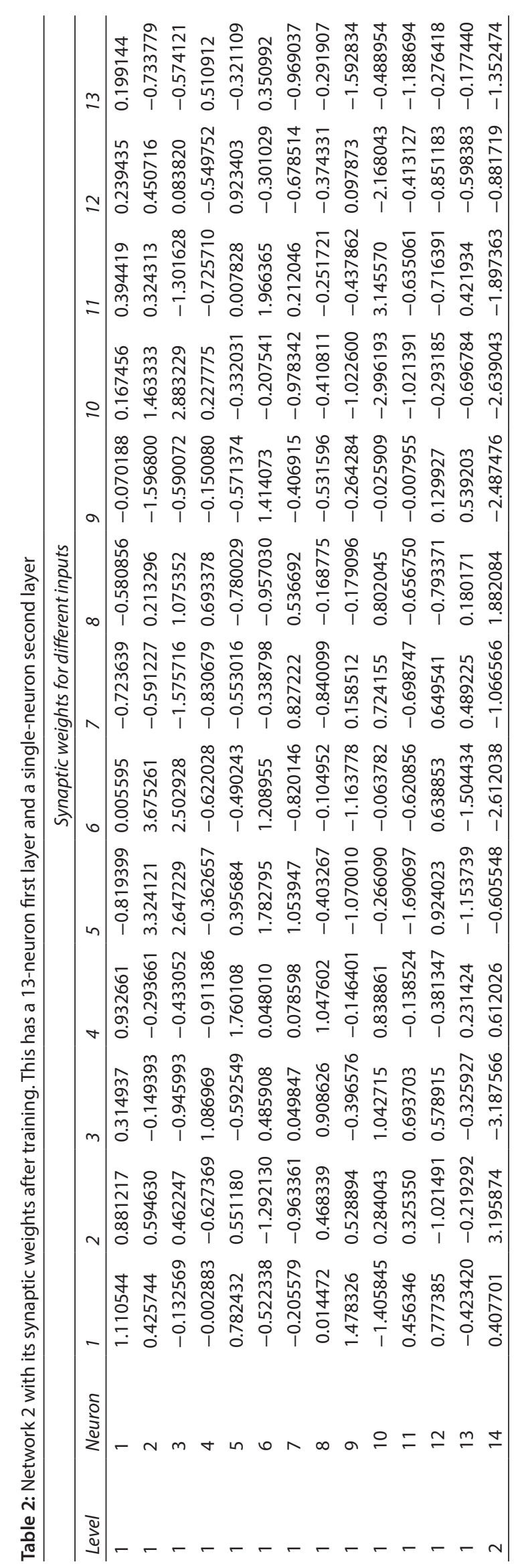


Table 3: Accuracy of prediction of overhanging dental restorations (ODRs) in the two networks and when accepting a \pm 1 error in the numbers of ODRs

\begin{tabular}{|c|c|c|c|c|c|c|c|}
\hline \multicolumn{4}{|l|}{ Network 1} & \multicolumn{4}{|c|}{ Network 2 (SCLs not included as input) } \\
\hline \multicolumn{2}{|c|}{$\begin{array}{l}\text { Accuracy predicting first } \\
100 \text { cases }\end{array}$} & \multirow{2}{*}{$\begin{array}{l}\text { Accuracy } \\
\text { predicting } \\
\text { undisclosed } \\
402 \text { cases } \\
\text { After training }\end{array}$} & \multirow{2}{*}{$\begin{array}{l}\text { Allowing } \\
\pm 1 \text { error in } \\
\text { prediction } \\
\text { (402 cases) } \\
\text { After training }\end{array}$} & \multicolumn{2}{|c|}{$\begin{array}{l}\text { Accuracy predicting first } \\
100 \text { cases }\end{array}$} & \multirow[t]{2}{*}{$\begin{array}{l}\text { Accuracy predict- } \\
\text { ing undisclosed } \\
402 \text { cases }\end{array}$} & \multirow{2}{*}{$\begin{array}{l}\text { Allowing } \\
\pm 1 \text { error in } \\
\text { prediction } \\
\text { (402 cases) } \\
\text { After training }\end{array}$} \\
\hline Before training & After training & & & Before training & After training & & \\
\hline $61 \%$ & $86 \%$ & $60.2 \%$ & $85.6 \%$ & $62 \%$ & $86 \%$ & $60.7 \%$ & $82.1 \%$ \\
\hline
\end{tabular}

SCLs, secondary caries lesions

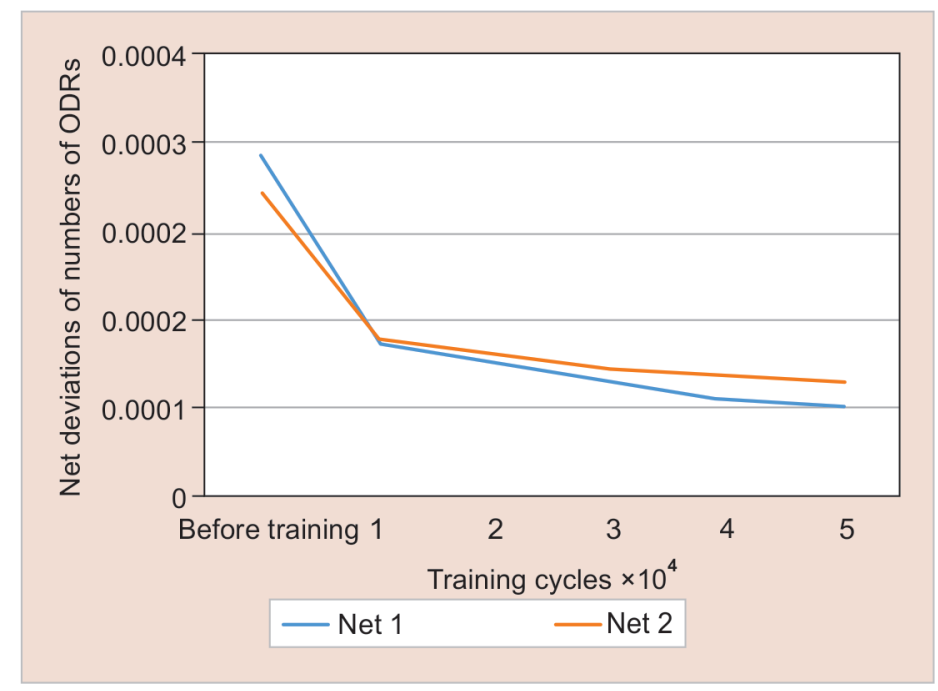

Fig. 2: Chart showing average deviations between net output and actual output of networks 1 and 2 for overhanging dental restoration predictions after every 10,000 training cycles

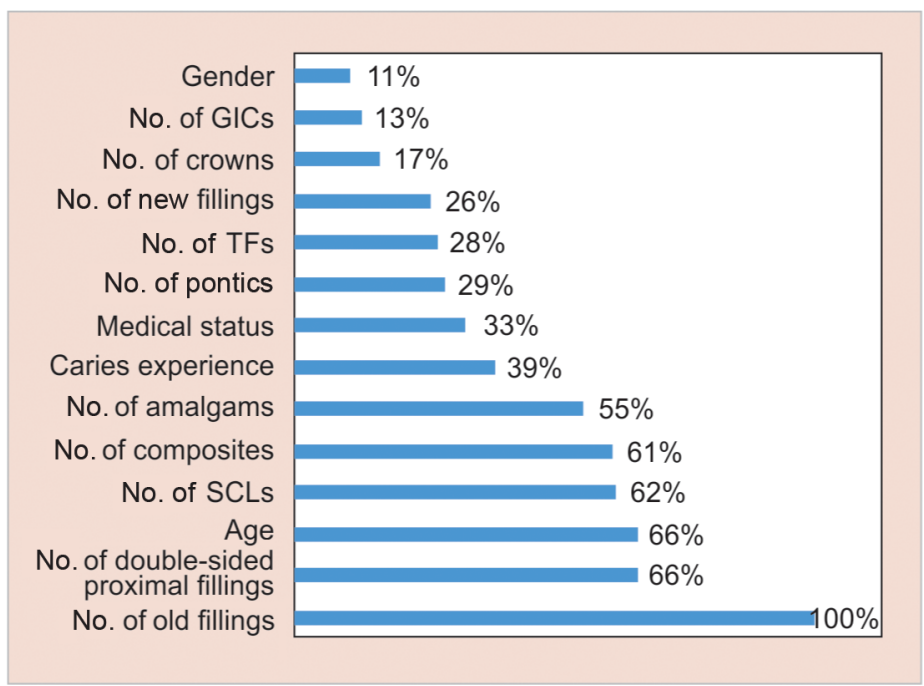

Fig. 3: Bar chart showing the relative/normalized independent factor importance in network 1

information was not collected in the current investigation; thus, such speculations would need future confirmation.

When adding SCLs as an input variable, the ANN model demonstrated a higher prediction accuracy for ODRs. This may be explained by the fact that SCLs lead to undermining of the tooth filling, where a combination of a caries lesion and an open margin is actually present. ${ }^{21}$ This would ultimately form a plaque retentive area, even though was not initially a true ODR. Accordingly, early management of secondary caries and underlying causes is considered a priority and should not be overlooked.

In a previous study by Ghulam and Fadel, ${ }^{22}$ individuals suffering from one or more medical conditions were observed to have more ODRs, whereas in the current investigation, medical status was of low input importance. This is explained by the fact that the previously used cluster analysis aimed to explain the observed distribution of current ODRs to direct interventional efforts and only utilized the few available data accordingly. In the present 
investigation, however, the formulated ANN models were meant to focus on future ODRs for preventive programs rather than prevalent ones and consequently required the utilization of a larger number of patient- and restoration-related input data.

The average deviation of the two ANN models was clearly reduced with the increasing number of training cycles. This was in line with the study by Xie et al., which utilized ANN modeling to determine the necessity for tooth extraction prior to orthodontic treatment. ${ }^{23}$ In that study, the prediction accuracy increased from 80 to $100 \%$ following effective pretraining.

Allowing for a marginal error of \pm 1 ODR increased the accuracy of the constructed ANN models, although this in itself may be considered as a study limitation as it allows for a degree of uncertainty. However, the subject of ODRs detection remains a highly subjective matter and is dependent on the used methods and settings. ${ }^{6}$ Moreover, this points to the fact that the current investigation only involved radiographic evaluation and was not complemented with a clinical examination, possibly affecting the noted prevalence of ODRs. ${ }^{6}$ Nevertheless, utilizing radiographs to construct the proposed prediction models may prove to be of significant importance and practicality in large surveillance studies, especially when detailed clinical examination is not possible.

\section{Conclusion}

Within the limitations of this study, it can be concluded that ANN modeling predicted the occurrence of ODRs with more than $85 \%$ accuracy. The most important predictive factor was the number of old fillings, while the least important factor was gender.

\section{Clinical Significance}

This study is the first to utilize a mathematical model using ANN in an attempt to predict the occurrence of ODRs. Making use of such analyses can help periodontists and general dentists predict occurrence of these clinical situations, formulate effective treatment planning, and reduce patient discomfort and unnecessary costs.

\section{Acknowledgments}

The authors express their gratitude to the administration of Prince Mohammed Bin Abdulaziz Hospital and staff of the dental department for their cooperation and support during the study.

\section{References}

1. Jokstad A, Bayne S, Blunck U, et al. Quality of dental restorations. FDI Commission Project 2-95. Int Dent J 2001;51(3):117-158. DOI: 10.1002/j.1875-595x.2001.tb00832.x.

2. Selwitz RH, Ismail Al, Pitts NB. Dental caries. Lancet 2007;369(9555): 51-59. DOI: 10.1016/S0140-6736(07)60031-2.

3. Fernandes N, Vally Z, Sykes L. The longevity of restorations -A literature review. South African Dent J 2015;70:410-413.

4. Donovan TE. Longevity of the tooth/restoration complex: a review. J California Dent Assoc 2006;34(2):122-128.

5. Sirajuddin S, Narasappa KM, Gundapaneni V, et al. latrogenic damage to periodontium by restorative treatment procedures: an overview. Open Dent J 2015;9(1):217-222. DOI: 10.2174/1874210601509010217.
6. Brunsvold MA, Lane JJ. The prevalence of overhanging dental restorations and their relationship to periodontal disease. J Clin Periodontol 1990;17(2):67-72. DOI: 10.1111/j.1600-051x.1990. tb01064.x.

7. Winter C, Payet JP, Suttle CA. Modeling the winter-to-summer transition of prokaryotic and viral abundance in the arctic ocean. PLoS ONE 2012;7(12):e52794-e. DOI: 10.1371/journal.pone. 0052794.

8. Gargouri N, Dammak Masmoudi A, Sellami Masmoudi D, et al. A new GLLD operator for mass detection in digital mammograms. Int J Biomed Imag 2012;2012:765649. DOI: 10.1155/2012/765649.

9. Kim EY, Lim KO, Rhee HS. Predictive modeling of dental pain using neural network. Stud Health Technol Informat 2009;146:745-746.

10. Dar-Odeh NS, Alsmadi OM, Bakri F, et al. Predicting recurrent aphthous ulceration using genetic algorithms-optimized neural networks. Adv Appl Bioinform Chem 2010;3:7-13. DOI: 10.2147/aabc. s10177.

11. Al Haidan A, Abu-Hammad O, Dar-Odeh N. Predicting tooth surface loss using genetic algorithms-optimized artificial neural networks. Computation Mathemat Methods Med 2014;2014:106236. DOI: 10.1155/2014/106236.

12. Fadel $\mathrm{H}, \mathrm{Al}$ Hamdan $\mathrm{K}$, Rhbeini $\mathrm{Y}$, et al. Root caries and risk profiles using the cariogram in different periodontal disease severity groups. Acta Odontol Scand 2011;69(2):118-124. DOI: 10.3109/00016357.2010.538718.

13. Lino JR, Ramos-Jorge J, Coelho VS, et al. Association and comparison between visual inspection and bitewing radiography for the detection of recurrent dental caries under restorations. Int Dent J 2015;65(4):178-181. DOI: 10.1111/idj.12172.

14. Pythia - The Neural Network Designer. USA: Runtime Software 2000.

15. Park WJ, Park J-B. History and application of artificial neural networks in dentistry. Europ J Dent 2018;12(4):594-601. DOI: 10.4103/ejd. ejd_325_18.

16. Sarkar NK. Creep, corrosion and marginal fracture of dental amalgams. J Oral Rehabilitat 1978;5(4):413-423. DOI: 10.1111/j.13652842.1978.tb01260.x.

17. Hervas-Garcia A, Martinez-Lozano MA, Cabanes-Vila J, et al. Composite resins. A review of the materials and clinical indications. Med Oral Patol Oral Cir Bucal 2006;11(2):E215-E220.

18. Herman K, Czajczyńska-Waszkiewicz A, Kowalczyk-Zając M, et al. Assessment of the influence of vegetarian diet on the occurrence of erosive and abrasive cavities in hard tooth tissues. Postepy Hig Med Dosw (Online) 2011;65:764-769. DOI: 10.5604/17322693.967066.

19. Staufenbiel I, Adam K, Deac A, et al. Influence of fruit consumption and fluoride application on the prevalence of caries and erosion in vegetarians--a controlled clinical trial. Europ J Clin Nutri 2015;69(10):1156-1160. DOI: 10.1038/ejcn.2015.20.

20. Moradi-Lakeh M, El Bcheraoui C, Afshin A, et al. Diet in Saudi Arabia: findings from a nationally representative survey. Pub Heal Nutri 2017;20(6):1075-1081. DOI: 10.1017/S1368980016003141.

21. Kirsch J, Tchorz J, Hellwig E, et al. Decision criteria for replacement of fillings: a retrospective study. Clin Experiment Dent Res 2016;2(2):121128. DOI: $10.1002 / \mathrm{cre} 2.30$

22. Ghulam OA, Fadel HT. Can clusters based on caries experience and medical status explain the distribution of overhanging dental restorations and recurrent caries? A cross-sectional study in Madinah - Saudi Arabia. Saudi J Biolog Sci 2018;25(2):367-371. DOI: 10.1016/j. sjbs.2017.02.001.

23. Xie X, Wang L, Wang A. Artificial neural network modeling for deciding if extractions are necessary prior to orthodontic treatment. Angle Orthod 2010;80(2):262-266. DOI: 10.2319/111608-588.1. 